\title{
Factores sociodemográficos y ambientales asociados con la actividad física deportiva en la población urbana del Perú
}

\author{
Juan A. Seclén-Palacín ${ }^{1}$ y Enrique R. Jacoby ${ }^{2}$
}

RESUMEN Objetivos. Determinar la frecuencia de la actividad deportiva en la población urbana del Perú e identificar los factores sociodemográficos, económicos y ambientales asociados con ella. Métodos. Se utilizó la información recogida por la Encuesta Nacional de Hogares del segundo trimestre del año 1997, Perú (ENAHO 97-II), a cargo del Instituto Nacional de Estadística e Informática del Perú. ENAHO-97 se basó en un muestreo probabilístico, multietápico y estratificado de todo el territorio nacional urbano dividido en ocho regiones geográficas: Lima metropolitana, costa norte, costa centro, costa sur, sierra norte, sierra centro, sierra sur y selva. En total se visitaron 14913 hogares y fueron entrevistadas 45319 personas de 15 años o mayores. La información sobre la frecuencia de la práctica deportiva fue clasificada en diaria, interdiaria (en días alternos), semanal u ocasional. La actividad deportiva regular (ADR) se definió como la práctica de cualquier deporte con una frecuencia diaria o interdiaria. Además se examinaron las preferencias y obstáculos para la práctica deportiva. Se realizó un análisis descriptivo de los niveles de ADR según el sexo mediante la prueba de ji al cuadrado. Se analizaron los factores asociados con la ADR mediante regresión logística múltiple condicional y análisis de residuos, multicolinearidad e interacciones. El nivel de significación utilizado fue de 0,05.

Resultados. La práctica de deportes al menos una vez a la semana y de ADR fue mayor entre los hombres (44,5 y 12,8\%, respectivamente) que ente las mujeres (32,4 y 10,5\%). El grupo de edad en que hubo una mayor práctica de ADR fue el de 50-55 años en los hombres (20\%) y el de 40-45 años en las mujeres (18\%). Se encontró que en la zona de la selva, sierra centro y costa centro se practica más deporte $(15,3,12,8$ y 12,1\%, respectivamente), mientras que en Lima metropolitana y sierra sur se observan los valores más bajos $(10,6$ y 9,7\%, respectivamente). El nivel de ingresos no mostró asociación con la práctica de $A D R$, aunque otras variables asociadas indirectamente con el nivel socioeconómico —como el mayor nivel educacional, tener empleo y tener acceso a Internet o a televisión por cable - y el consumo de información deportiva estuvieron significativa y directamente asociados con la práctica de ADR. Las barreras más frecuentes para la práctica del deporte fueron la falta de tiempo y de infraestructura y el desinterés de la población. La práctica de ADR por parte de los miembros de un hogar estuvo significativamente asociada con la presencia de un jefe de hogar que practicaba deportes activamente.

Conclusiones. La práctica de ADR en las zonas urbanas del Perú es escasa. Los más afectados son los menores de 30 años, las mujeres y los residentes del área metropolitana de la capital. Esta insuficiente práctica de ADR constituye un reto para la salud pública y reafirma la necesidad de promover estilos de vida activos. La influencia positiva observada cuando el jefe del hogar es un deportista activo y la mayor ADR encontrada en zonas urbanas fuera del área metropolitana de Lima deben ser objeto de estudios más profundos y deben tomarse en consideración para el diseño de intervenciones específicas.

Palabras clave Actividad física, deportes, estilo de vida, Perú.

1 Organización Panamericana de la Salud, Proyecto Desarrollo de Sistemas y Servicios de Salud. La correspondencia debe ser dirigida a Juan A. SeclénPalacín, Representación de la OPS en Brasil, Setor
Embaixada Norte Lote 19, CEP 70800-400, Brasilia, Distrito Federal, Brasil. Correo electrónico: seclenj@ bra.ops-oms.org.
2 Organización Panamericana de la Salud, Programa de Alimentos y Nutrición, Washington D.C. Correo electrónico: jacobyen@paho.org. 
De acuerdo con la Organización Mundial de la Salud, el sedentarismo es uno de los factores de riesgo más importantes de sufrir enfermedades crónicas no transmisibles, junto con la alimentación inadecuada y el tabaquismo. La obesidad, la diabetes, las enfermedades del corazón y algunos cánceres se asocian con $40 \%$ de la morbilidad y $60 \%$ de la mortalidad general en el mundo y han dejado de ser problemas exclusivos de las sociedades de mayor poderío económico (1).

Cerca de dos tercios de la población mundial mantiene prácticas sedentarias como resultado de la urbanización, que trae consigo el predominio de trabajos con bajo gasto energético, un mayor uso de transportes motorizados y un mayor empleo del tiempo libre en actividades sedentarias, como mirar televisión (1). Por ejemplo, en las zonas urbanas del Perú, cerca de tres cuartas partes de los trabajadores de uno $u$ otro sexo están empleados en actividades básicamente sedentarias.

En América Latina y el Caribe no se cuenta con suficiente información acerca del sedentarismo y sus causas. Está documentado que el sobrepeso ${ }^{3}$ afecta a cerca de la mitad de la población adulta $(2,3)$ y se observa un aumento de la incidencia de enfermedades no transmisibles (4). Como se sabe, una de las causas fundamentales, tanto del aumento de la prevalencia de estas enfermedades como del sobrepeso, es el incremento del sedentarismo, que se considera uno de los cinco factores de riesgo más importantes para la salud (5). Según los resultados de un estudio realizado en siete ciudades de América Latina, 65\% de los adultos mayores realizan poca actividad física (6).

El Perú no es una excepción a esta realidad. El sobrepeso empezó a manifestarse como fenómeno social a inicios de la década de $1990(7,8)$ y se ha extendido rápidamente hasta abarcar a dos terceras partes de la población adulta (9). Sin embargo, aún no se

\footnotetext{
3 El sobrepeso se define como un índice de masa corporal $\geq 25$. El índice de masa corporal es la razón dada por el peso en kilogramos dividido por el cuadrado de la estatura en metros.
}

dispone de información sobre los niveles de inactividad física de los distintos grupos de la población peruana.

Estudios epidemiológicos han demostrado la existencia de una relación directa entre la inactividad física y diversas enfermedades crónicas e incluso la muerte prematura (11). Se ha demostrado también el efecto protector de la actividad física regular contra las cardiopatías isquémicas $(12,13)$, la diabetes mellitus $(14,15)$, los trastornos cerebrovasculares (16), la obesidad (17), la osteoporosis (18), la ansiedad y la depresión (19), y algunos tipos de cáncer (20). Para corroborar dicho efecto, diversos estudios experimentales han demostrado los beneficios de la actividad física en relación con algunos factores de riesgo, como la hipertensión, el perfil lipídico, la tolerancia a la glucosa, la densidad ósea, etc. (21).

De estos trabajos surgió durante las décadas de 1970 y 1980 el concepto de fitness (correspondiente a "buena condición física"), con un hincapié especial en mejorar la capacidad cardiorrespiratoria mediante ejercicios como medio para lograr beneficios para la salud. Este concepto se tradujo en la recomendación de realizar al menos tres sesiones semanales de ejercicios, con una duración mínima de 30 minutos cada una y una intensidad equivalente a 6090\% del pulso cardíaco máximo (11).

En la década de 1990, sin embargo, se demostró que la cantidad de ejercicio necesaria para producir beneficios de salud es menor que la requerida para alcanzar una condición física óptima. En este sentido, se recomienda practicar 30 minutos diarios de actividad física moderada - como caminar, bailar, subir escaleras o montar bicicletapor lo menos cinco veces a la semana. Según esta recomendación, los 30 minutos de este tipo de actividad no necesitan realizarse en una sola sesión, pues pueden acumularse durante el día en tandas de 10 minutos cada una (11).

De lo anterior puede concluirse que mediante la evaluación de la práctica de deportes de una población se puede hacer un cálculo aproximado de su actividad física, específicamente de aquella que es capaz de producir beneficios de salud. El presente estudio tiene como objetivo determinar la frecuencia de la actividad deportiva en la población urbana del Perú e identificar los factores sociodemográficos, económicos y ambientales asociados con ella.

\section{MÉTODOS}

\section{Población de estudio}

El Perú tiene una población de $26 \mathrm{mi}-$ llones de habitantes, de los cuales $65 \%$ viven en zonas urbanas de 2000 habitantes o más. Cincuenta y cuatro por ciento de la población total se considera pobre y el porcentaje de analfabetismo en mayores de 15 años es de $7 \%$. Lima, la capital, está situada en el litoral suroeste del Océano Pacífico peruano y tiene 7,4 millones de habitantes (22).

\section{Selección de la muestra}

Para este estudio se utilizó la información recogida por la Encuesta Nacional de Hogares del segundo trimestre del año 1997, Perú (ENAHO 97-II), a cargo del Instituto Nacional de Estadística e Informática del Perú (INEI). La encuesta abarcó todo el territorio nacional urbano dividido en ocho regiones geográficas: Lima metropolitana, costa norte, costa centro, costa sur, sierra norte, sierra centro, sierra sur y selva.

ENAHO-97 se basó en un muestreo probabilístico, multietápico, estratificado por áreas geográficas. Las unidades primarias de muestreo fueron los centros poblados urbanos ( $\geq 2000$ habitantes). A continuación se realizó el muestreo por conglomerados (aproximadamente 100 viviendas particulares) y como unidades terciarias de muestreo se tomaron las viviendas particulares.

En total se visitaron 14913 hogares y se entrevistó a 45319 personas de 15 años o mayores. La recolección de datos estuvo a cargo de encuestadores entrenados en el uso y aplicación del cuestionario. El cuestionario de ENAHO estaba estructurado en $12 \mathrm{sec}-$ ciones, de las cuales 4 (características de la vivienda y del hogar, informa- 
ción sobre sus miembros y práctica de deportes) se utilizaron para el presente estudio.

La sección sobre los deportes estaba dirigida a explorar la actividad deportiva de los entrevistados, a los que se les preguntó: “¿En el último mes, usted o algún miembro de su hogar practicó deportes?" Si la respuesta era positiva, se indagaba acerca de la frecuencia con que cada miembro del hogar practicaba el deporte. Las respuestas fueron clasificadas por el encuestador en una de las siguientes categorías: diaria, interdiaria (en días alternos), semanal $\mathrm{u}$ ocasional. Para conocer el tipo de deporte practicado se preguntó "¿Qué tipo de deporte practica usted mayormente?"

Se indagó sobre la percepción del jefe del hogar acerca de la infraestructura deportiva en el vecindario, las barreras que dificultan que la población practique deportes y los tipos de deporte que se practican al aire libre en su comunidad.

\section{Variables estudiadas}

Se creó la variable "actividad deportiva regular" (ADR), definida como la práctica de cualquier deporte o actividad física, incluida la de caminar, con una frecuencia diaria o interdiaria durante el mes previo a la entrevista. Mediante esta variable se identificó a las personas que realizaban la cantidad mínima de actividad capaz de brindar beneficios de salud. ${ }^{4}$

En relación con el ámbito geográfico se consideraron dos categorías: Lima metropolitana y resto urbano. Esta última se definió como toda población con más de 2000 habitantes ubicada fuera del área metropolitana de la capital.

Para establecer el nivel económico se tomó en cuenta el ingreso mensual per cápita del hogar. Para esto se dividió el ingreso familiar mensual entre el número total de miembros del hogar mayores de 15 años. Este valor fue pos-

\footnotetext{
4 Tres sesiones semanales de ejercicios, de un mínimo de 30 minutos cada una, con una intensidad equivalente a $60-90 \%$ del pulso cardiaco máximo.
}

CUADRO 1. Características generales de la población estudiada ( $n=45319$ )

\begin{tabular}{|c|c|c|}
\hline Característica & No. & $\%$ \\
\hline \multicolumn{3}{|l|}{ Sexo } \\
\hline Mujeres & 23521 & 51,9 \\
\hline Hombres & 21798 & 48,1 \\
\hline \multicolumn{3}{|l|}{ Grupo de edad (años) } \\
\hline $15-19$ & 6604 & 14,6 \\
\hline $20-29$ & 12615 & 27,8 \\
\hline $30-44$ & 13273 & 29,3 \\
\hline $45-59$ & 7648 & 16,9 \\
\hline 60 a más & 5179 & 11,4 \\
\hline \multicolumn{3}{|l|}{ Ámbito geográfico } \\
\hline Urbano (Lima metropolitana) & 6349 & 14,0 \\
\hline Resto urbano & 38970 & 86,0 \\
\hline \multicolumn{3}{|l|}{ Nivel educacional } \\
\hline Primaria o menor & 13730 & 30,3 \\
\hline Secundaria & 18687 & 41,2 \\
\hline Superior & 12902 & 28,5 \\
\hline \multicolumn{3}{|l|}{ Situación civil } \\
\hline No unido (soltero, divorciado, viudo, separado) & 20610 & 45,5 \\
\hline Unido (casado, conviviente) & 24709 & 54,5 \\
\hline \multicolumn{3}{|l|}{ Ingresos económicos } \\
\hline Bajos ingresos & 17673 & 39,0 \\
\hline Medianos ingresos & 19109 & 42,2 \\
\hline Altos ingresos & 8537 & 18,8 \\
\hline \multicolumn{3}{|l|}{ Acceso a tecnología de comunicación } \\
\hline Acceso a televisión por cable & 1826 & 4,0 \\
\hline Acceso al Internet en domicilio & 112 & 0,2 \\
\hline Sin acceso a televisión por cable ni Internet en domicilio & 43462 & 95,8 \\
\hline \multicolumn{3}{|l|}{ Consumo de información deportiva } \\
\hline Radioescucha de programa deportivo & 28386 & 62,6 \\
\hline Televidente deportivo & 37089 & 81,8 \\
\hline Lector deportivo & 4587 & 10,1 \\
\hline No consume información deportiva & 4786 & 10,6 \\
\hline
\end{tabular}

teriormente deflactado ${ }^{5}$ y estratificado en quintiles de ingreso, desde el quintil I (menor ingreso económico) hasta el quintil V (mayores ingresos). Con el fin de simplificar el análisis, posteriormente se reagrupó esta variable en tres estratos según los ingresos: alto (quintil V), mediano (quintiles III y IV) y bajo (quintiles I y II).

\section{Análisis estadístico}

Las frecuencias simples de las variables sexo, nivel educacional, grupo de edad, ingreso familiar mensual y ámbito geográfico se calcularon y relacionaron con la variable depen-

\footnotetext{
5 La deflactación es un procedimiento de estandarización económica de los ingresos y precios de la canasta básica de alimentos según la región geográfica, con el fin de permitir comparaciones entre regiones.
}

diente ADR mediante la prueba de ji al cuadrado.

Con el fin de examinar los factores sociodemográficos, económicos y ambientales asociados con la ADR se elaboró un modelo explicatorio basado en las variables demográficas y sociales disponibles en la encuesta. Para ello se realizó un análisis de regresión logística múltiple condicional y un análisis de residuos, multicolinearidad e interacciones. En este análisis se tomaron en cuenta solo los mayores de 17 años. Se utilizó el paquete estadístico Statistical Package for Social Sciences (SPSS) versión 10.1. El nivel de significación utilizado fue de 0,05.

\section{RESULTADOS}

Las características generales de la población estudiada se muestran en el cuadro 1. De las 45319 personas entre- 
CUADRO 2. Porcentaje de personas que practican con regularidad una actividad deportiva, según el sexo, en la población urbana del Perú

\begin{tabular}{|c|c|c|c|c|c|c|}
\hline \multirow[b]{2}{*}{ Frecuencia } & \multicolumn{2}{|c|}{ Hombres $(n=21798)$} & \multicolumn{2}{|c|}{ Mujeres $(n=23521)$} & \multicolumn{2}{|c|}{ Total $(n=45319)$} \\
\hline & $\%$ & IC95\% ${ }^{a}$ & $\%$ & IC95\% & $\%$ & IC95\% \\
\hline Diaria & 8,2 & $8,15-8,21$ & 7,0 & $6,99-7,04$ & 7,6 & $7,52-7,63$ \\
\hline Interdiaria ${ }^{\mathrm{b}}$ & 4,6 & $4,54-4,69$ & 3,5 & $3,41-3,59$ & 4,0 & $3,94-4,11$ \\
\hline Semanal & 31,7 & $31,67-31,74$ & 21,9 & $21,87-21,92$ & 26,6 & $26,59-26,67$ \\
\hline Ocasional & 10,2 & $10,15-10,23$ & 6,2 & $6,17-6,21$ & 8,1 & $8,07-8,16$ \\
\hline No practica & & & & & & \\
\hline deportes & 45,3 & $45,26-45,71$ & 61,4 & $61,1-61,7$ & 53,7 & $53,61-53,69$ \\
\hline
\end{tabular}

a IC95\%: intervalo de confianza de 95\%.

${ }^{\mathrm{b}}$ En días alternos.

FIGURA 1. Porcentaje de personas que practican con regularidad una actividad deportiva en la población urbana del Perú, según edad y sexo $(n=45319)$

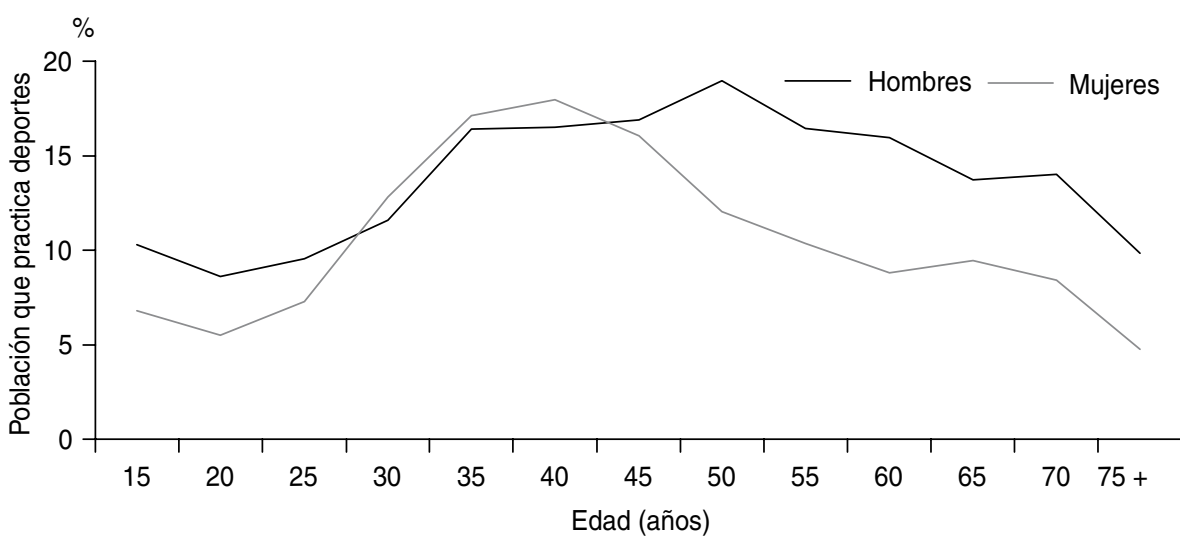

CUADRO 3. Frecuencia porcentual de la práctica deportiva, según grupo de edad y sexo, en la población urbana del Perú

\begin{tabular}{lccccrr}
\hline & \multicolumn{5}{c}{ Grupo de edad (años) } \\
\cline { 2 - 7 } \multicolumn{1}{c}{ Frecuencia } & \begin{tabular}{l} 
15-19 \\
\multicolumn{1}{c}{$\%$}
\end{tabular} & $\begin{array}{c}20-29 \\
\%\end{array}$ & $\begin{array}{c}30-44 \\
\%\end{array}$ & $\begin{array}{c}45-59 \\
\%\end{array}$ & $\begin{array}{c}\geq 60 \\
\%\end{array}$ & Total \\
\hline & & & Hombres & $(n=21798)$ \\
Diaria & 7,0 & 5,6 & 9,2 & 10,7 & 9,9 & 8,2 \\
Interdiaria & 3,3 & 3,5 & 5,5 & 6,8 & 3,6 & 4,6 \\
Semanal & 21,2 & 24,2 & 38,5 & 44,0 & 28,2 & 31,7 \\
Ocasional & 2,6 & 6,7 & 14,3 & 15,3 & 10,6 & 10,2 \\
No practica deportes & 65,9 & 60,0 & 32,5 & 23,2 & 47,7 & 45,3 \\
& & & Mujeres & $(n=23521)$ & & \\
Diaria & 4,5 & 4,2 & 10,4 & 9,1 & 5,2 & 7,0 \\
Interdiaria & 2,2 & 2,1 & 5,4 & 4,2 & 2,6 & 3,5 \\
Semanal & 13,1 & 13,7 & 30,8 & 31,7 & 15,6 & 21,9 \\
Ocasional & 2,1 & 5,3 & 8,0 & 8,8 & 4,9 & 6,2 \\
No practica deportes & 78,1 & 74,7 & 45,4 & 46,2 & 71,7 & 61,4 \\
\hline
\end{tabular}

vistadas, 51,9\% eran mujeres y $14 \%$ eran residentes del área metropolitana de Lima. Aproximadamente 15\% eran jóvenes menores de 19 años y 29,3\% te- nían entre 30 y 44 años de edad. De los entrevistados, $28,5 \%$ tenían una educación superior y 18,8\% pertenecían al nivel socioeconómico superior. La pro- porción de entrevistados con acceso a televisión por cable e Internet fue muy pequeña ( 4,0 y $0,2 \%$, respectivamente).

\section{Actividad deportiva regular}

Más de la tercera parte $(38,2 \%)$ de la población entrevistada practicaba deportes o ejercicios al menos una vez a la semana, y esta proporción fue mayor entre los hombres (44,5\%) que entre las mujeres $(32,4 \%)$ (cuadro 2 ). Por otro lado, $11,6 \%$ de las personas practicaban ADR con frecuencia diaria o interdiaria, que también fue ligeramente mayor en hombres $(12,8 \%)$ que en mujeres (10,5\%). El grupo de edad que practicaba más ADR fue el de 50 55 años en los hombres (20\%) y el de 40-45 años en las mujeres (18\%). En los restantes grupos de edad, la proporción de personas que practicaban ADR fue menor en ambos sexos, pero particularmente entre las mujeres (figura 1). Por otro lado, la proporción de personas que practicaban deportes una vez por semana en el período entre la adolescencia y los 60 años de edad fue dos veces mayor (de 21,2\% a $44 \%$ ) entre los hombres y casi tres veces mayor (de $13,1 \%$ a $31,7 \%$ ) entre las mujeres de los mismos grupos de edad (cuadro 3).

Como se puede observar en la figura 2, las ADR no se practicaban de manera homogénea en las regiones geográficas estudiadas; así, en la zona de la selva, sierra centro y costa centro se practicaba más deporte $(15,3,12,8$ y $12,1 \%$, respectivamente), mientras que en Lima metropolitana y sierra sur se observaron los valores más bajos $(10,6$ y $9,7 \%$, respectivamente).

Cuando se comparó la práctica de ADR en hombres y mujeres según diversas características sociodemográficas y económicas, se observó que la ADR fue significativamente mayor en hombres que en mujeres en todos los casos (cuadro 4). El nivel socioeconómico no mostró relación con la ADR, pero el nivel educacional muestra una asociación directa con ella, aunque solo entre los hombres. Es importante anotar también que tanto los hombres como las mujeres de Lima metropolitana 
FIGURA 2. Porcentaje de la población urbana que practica con regularidad una actividad deportiva en el Perú, según regiones geográficas $(n=45319)$

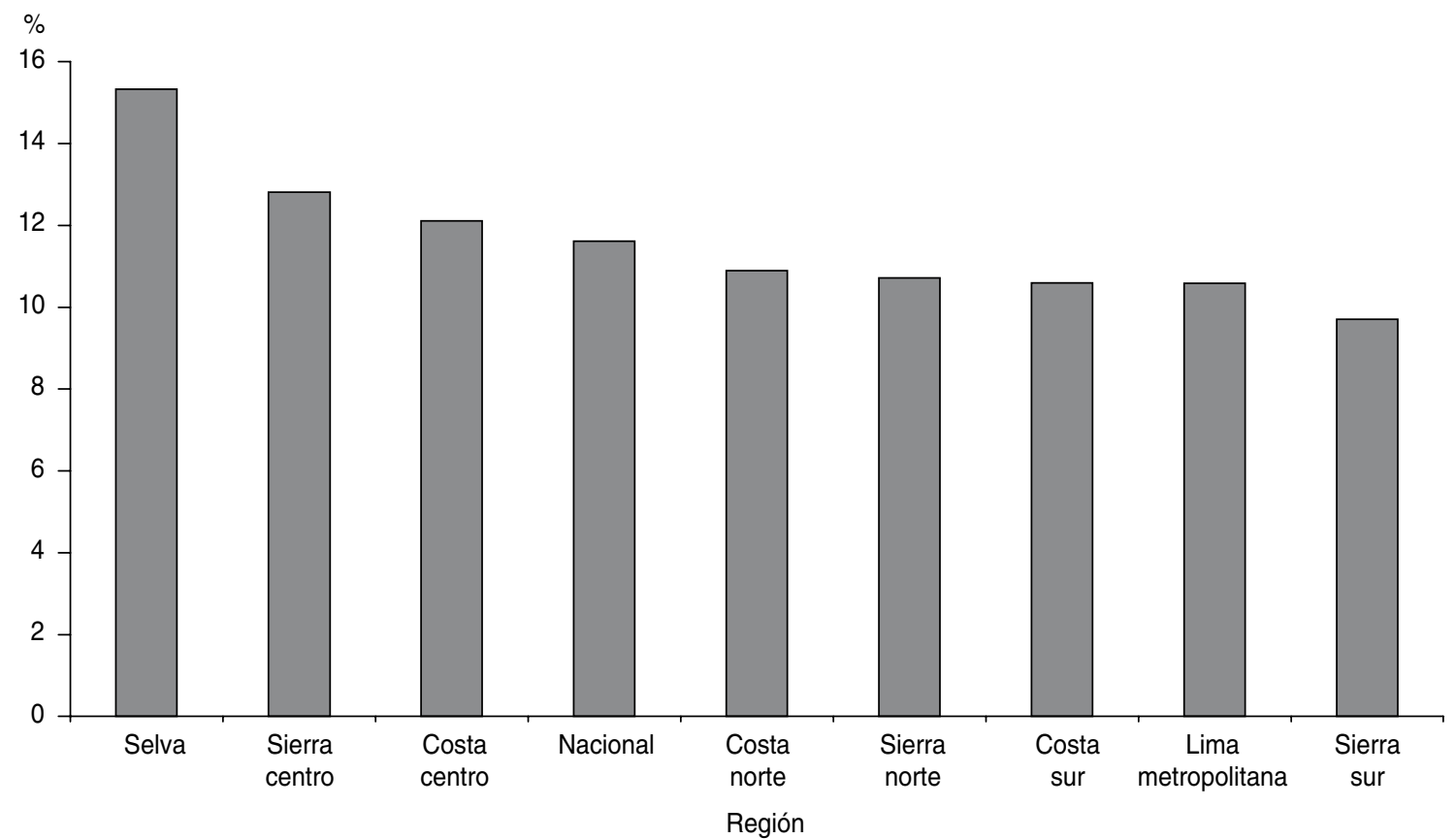

practicaban menos ADR que las personas que vivían en zonas urbanas fuera del área metropolitana de la capital.

\section{Preferencias deportivas}

En cuanto a las preferencias de la población en general, el fútbol y el fulbito (fútbol practicado en un campo pequeño), en su conjunto, constituyeron la categoría deportiva de mayor aceptación entre los hombres (63\%), mientras que las mujeres prefirieron el voleibol (54.5\%). El fútbol/fulbito gozaba de similar popularidad en todos los grupos de edad, mientras que el voleibol y el baloncesto fueron los deportes preferidos por los adolescentes y los adultos jóvenes. Los grupos de mayor edad preferían algunos deportes de menor impacto, como el atletismo, los ejercicios aeróbicos o trotar (cuadro 5). Por otro lado, el fútbol/ fulbito y el voleibol resultaron ser más populares entre los estratos de menores ingresos (fútbol: coeficiente de Spearman $r=-0,9, P=0,036$ ), mientras que las opciones deportivas de las per-

CUADRO 4. Porcentaje de la población que practica con regularidad una actividad deportiva en zonas urbanas del Perú, según sexo y algunos factores sociodemográficos y económicos $(n=45319)$

\begin{tabular}{lccc}
\hline \multicolumn{1}{c}{ Estrato } & $\begin{array}{c}\text { Hombres } \\
\%\end{array}$ & $\begin{array}{c}\text { Mujeres } \\
\%\end{array}$ & $P^{\mathrm{a}}$ \\
\hline Población estudiada & 12,8 & 10,5 & $<0,001$ \\
Grupo de edad (años) & 10,3 & 6,8 & $<0,001$ \\
$15-19$ & 9,0 & 6,3 & $<0,001$ \\
$20-29$ & 14,7 & 15,8 & 0,08 \\
$30-44$ & 17,5 & 13,3 & $<0,001$ \\
$45-59$ & 13,5 & 7,8 & $<0,001$ \\
$\geq 60$ & 13,1 & 11,4 & 0,01 \\
Nivel educacional & 12,5 & 10,1 & $<0,001$ \\
$\quad$ Primaria o menor & 12,9 & 10,0 & $<0,001$ \\
$\quad$ Secundaria & 13,1 & 11,4 & $<0,001$ \\
$\quad$ Superior & 12,0 & 10,0 & $<0,001$ \\
Ingresos económicos & 14,2 & 9,9 & $<0,001$ \\
$\quad$ Bajos & & & $<0,001$ \\
Medianos & 11,4 & 9,9 & $<0,001$ \\
Altos & 13,0 & 10,6 & \\
Área de residencia & Lima metropolitana & & \\
Resto urbano & & & \\
\hline
\end{tabular}

a Prueba de ji al cuadrado, análisis de dos variables, nivel de significación de 0,05.

sonas de mayores ingresos fueron más diversas.

Aproximadamente la mitad de los jefes de hogar entrevistados informaron que había lugares adecuados para jugar al fútbol/fulbito en su vecindario; $15.5 \%$ sabía que había piscinas y $10,1 \%$, gimnasios (cuadro 6). Como las barreras más importantes para la práctica de los deportes los encuestados se- 
CUADRO 5. Porcentaje de personas que practican distintos tipos de deportes en zonas urbanas del Perú, según algunas características sociodemográficas $(n=21005)$

\begin{tabular}{|c|c|c|c|c|c|c|c|}
\hline Características & $\begin{array}{c}\text { Fútbol/fulbito } \\
\%\end{array}$ & $\begin{array}{c}\text { Voleibol } \\
\%\end{array}$ & $\begin{array}{c}\text { Baloncesto } \\
\%\end{array}$ & $\begin{array}{c}\text { Atletismo } \\
\%\end{array}$ & $\begin{array}{c}\text { Aeróbicos } \\
\%\end{array}$ & $\begin{array}{c}\text { Natación } \\
\%\end{array}$ & $\begin{array}{c}\text { Otros }^{a} \\
\%\end{array}$ \\
\hline \multicolumn{8}{|l|}{ Sexo } \\
\hline Mujeres & 25,0 & 54,5 & 5,7 & 2,1 & 2,3 & 0,4 & 10,0 \\
\hline Hombres & 63,0 & 16,6 & 4,5 & 2,4 & 1,1 & 0,7 & 11,7 \\
\hline \multicolumn{8}{|c|}{ Grupo de edad (años) } \\
\hline $15-19$ & 59,6 & 28,5 & 6,9 & 0,9 & 0,2 & 0,2 & 3,7 \\
\hline $20-29$ & 60,1 & 21,1 & 4,4 & 2,0 & 1,8 & 0,7 & 9,9 \\
\hline $30-44$ & 60,3 & 22,1 & 5,0 & 2,2 & 1,4 & 0,6 & 8,4 \\
\hline $45-59$ & 60,7 & 16,8 & 4,9 & 2,6 & 2,1 & 0,3 & 12,6 \\
\hline$\geq 60$ & 47,9 & 13,4 & 4,8 & 3,3 & 3,1 & 0,9 & 26,6 \\
\hline \multicolumn{8}{|c|}{ Ingresos económicos } \\
\hline Bajos & 66,0 & 24,6 & 3,5 & 1,4 & 0,4 & 0,3 & 3,8 \\
\hline Medianos & 59,7 & 19,7 & 5,8 & 2,6 & 1,7 & 0,4 & 10,1 \\
\hline Altos & 42,1 & 13,6 & 7,5 & 3,1 & 4,7 & 1,4 & 27,6 \\
\hline
\end{tabular}

${ }^{a}$ Artes marciales, tenis de mesa, tenis, bolos, entre otros.

ñalaron la falta de tiempo $(46,2 \%)$, el desinterés $(42,7 \%)$ y la falta de infraestructura deportiva (35\%).

\section{Análisis de la práctica deportiva}

Las regresiones logísticas calculadas para hombres y mujeres mayores de 17 años se presentan en el cuadro 7. La razón de posibilidades $(\mathrm{RP})$ calculada para cada factor o variable fue ajustada según las variables restantes del modelo. En comparación con el grupo de 17-29 años, entre los hombres se observó el incremento de la probabilidad de ADR a partir de los 30 años hasta alcanzar un máximo de 1,37 veces más en el grupo de 45-59 años. Entre las mujeres, la ADR al- canzó su frecuencia máxima entre los 30 y los 44 años de edad (2,3 veces más) y disminuyó en los grupos de edad subsiguientes.

Por otro lado, entre los hombres, la educación se asoció directamente con la ADR, mientras que el nivel socioeconómico pareció tener una asociación inversa con ella, aunque no significativa. En las mujeres, ni la edu-

CUADRO 6. Percepción del jefe del hogar sobre la disponibilidad de infraestructura deportiva y las barreras que limitan la práctica del deporte. ${ }^{a}$ Perú, zonas urbanas

\begin{tabular}{|c|c|c|c|c|c|}
\hline Característica & $\begin{array}{c}\text { Hombres } \\
(n=7504) \\
\%\end{array}$ & $\begin{array}{c}\text { Mujeres } \\
(n=7109) \\
\%\end{array}$ & $\begin{array}{c}\text { Lima } \\
\text { metropolitana } \\
(n=1890) \\
\%\end{array}$ & $\begin{array}{c}\text { Resto } \\
\text { urbano } \\
(n=12708) \\
\%\end{array}$ & $\begin{array}{c}\text { Total } \\
(n=14598) \\
\%\end{array}$ \\
\hline \multicolumn{6}{|c|}{$\begin{array}{l}\text { Percepción sobre disponibilidad } \\
\text { de infraestructura deportiva en el vecindario }\end{array}$} \\
\hline Cancha de fulbito & 56,9 & 52,3 & 56,9 & 54,3 & 54,6 \\
\hline Losa deportiva & 47,1 & 51,1 & 58,6 & 48,0 & 49,4 \\
\hline Cancha de fútbol & 40,6 & 38,9 & 38,6 & 39,9 & 39,8 \\
\hline Piscina & 15,6 & 15,5 & 22,9 & 14,4 & 15,5 \\
\hline Gimnasio & 10,1 & 11,6 & 28,8 & 8,2 & 10,8 \\
\hline Pista de atletismo & 4,5 & 4,2 & 9,8 & 3,5 & 4,3 \\
\hline Otros & 1,5 & 1,7 & 2,3 & 1,5 & 1,6 \\
\hline \multicolumn{6}{|l|}{ Barreras para la práctica deportiva } \\
\hline Falta de tiempo & 47,5 & 44,9 & 51,2 & 45,5 & 46,2 \\
\hline Desinterés/No gusta & 42,5 & 42,8 & 31,3 & 44,4 & 42,7 \\
\hline Falta de infraestructura deportiva & 37,2 & 32,7 & 32,6 & 35,4 & 35,0 \\
\hline Falta de motivación & 12,9 & 9,8 & 6,8 & 12,0 & 11,4 \\
\hline No hay organización de barrio & 2,3 & 1,6 & 1,5 & 2,0 & 2,0 \\
\hline Otros & 4,7 & 3,7 & 3,2 & 4,4 & 4,2 \\
\hline No sabe/No opina & 2,1 & 5,0 & 1,6 & 3,8 & 3,5 \\
\hline
\end{tabular}

a Porcentaje del número de respuestas. Cada informante proporcionó una respuesta o más. 
CUADRO 7. Regresión logística a de los factores sociodemográficos y económicos asociados con la práctica de actividad deportiva regular $(A D R)^{\mathrm{a}}$ en la población urbana del Perú $(n=45319)$

\begin{tabular}{|c|c|c|c|c|}
\hline \multirow[b]{2}{*}{ Factor estudiado } & \multicolumn{2}{|c|}{ Hombres } & \multicolumn{2}{|c|}{ Mujeres } \\
\hline & $\mathrm{RPa}(\mathrm{IC} 95 \%)^{\mathrm{b}}$ & $P$ & $\mathrm{RPa}(\mathrm{IC} 95 \%)$ & $P$ \\
\hline \multicolumn{5}{|l|}{ Grupo de edad (años) } \\
\hline $15-19$ & 1,00 & & 1,00 & \\
\hline 20-29 & $0,74(0,63-0,87)$ & 0,002 & $0,89(0,74-1,06)$ & 0,020 \\
\hline $30-44$ & $1,08(0,90-1,28)$ & 0,405 & $2,30(1,93-2,74)$ & $<0,001$ \\
\hline $45-59$ & $1,37(1,13-1,65)$ & $<0,001$ & $1,96(1,63-2,37)$ & $<0,001$ \\
\hline$\geq 60$ & $1,23(1,01-1,50)$ & 0,047 & $1,31(1,06-1,62)$ & 0,014 \\
\hline \multicolumn{5}{|l|}{ Ámbito geográfico } \\
\hline Resto urbano & 1,00 & & 1,00 & \\
\hline Lima metropolitana & $0,79(0,70-0,91)$ & $<0,001$ & $0,85(0,75-0,98)$ & 0,019 \\
\hline \multicolumn{5}{|l|}{ Nivel educacional } \\
\hline Superior & 1,00 & & 1,00 & \\
\hline Secundaria & $0,97(0,88-1,08)$ & 0,579 & $0,98(0,87-1,10)$ & 0,692 \\
\hline Primaria o menos & $0,85(0,75-0,97)$ & 0,016 & $0,92(0,81-1,05)$ & 0,222 \\
\hline \multicolumn{5}{|l|}{ Estado civil } \\
\hline Soltero, divorciado, viudo & 1,00 & & 1,00 & \\
\hline Unido (casado, conviviente) & $1,41(1,26-1,60)$ & $<0,001$ & $1,17(1,06-1,29)$ & 0,002 \\
\hline \multicolumn{5}{|l|}{ Ingresos económicos } \\
\hline Altos & 1,00 & & 1,00 & \\
\hline Medianos & $0,91(0,81-1,02)$ & 0,091 & $1,07(0,95-1,22)$ & 0,263 \\
\hline Bajos & $1,04(0,92-1,17)$ & 0,568 & $1,31(1,14-1,50)$ & $<0,001$ \\
\hline \multicolumn{5}{|l|}{ Situación laboral } \\
\hline Sin empleo & 1,00 & & 1,00 & \\
\hline Empleado & $1,15(1,03-1,29)$ & 0,019 & $1,12(1,02-1,22)$ & 0,016 \\
\hline \multicolumn{5}{|l|}{ Acceso a tecnologías de comunicación } \\
\hline Acceso a TV por cable & $1,62(1,34-1,98)$ & $<0,001$ & $1,40(1,13-1,73)$ & 0,002 \\
\hline Acceso a Internet en su domicilio & $1,17(0,55-2,51)$ & 0,679 & $2,21(1,16-4,19)$ & 0,015 \\
\hline \multicolumn{5}{|l|}{ Consumo de información deportiva } \\
\hline Radioaficionado al deporte & $1,03(0,94-1,12)$ & 0,519 & $1,14(1,04-1,25)$ & 0,004 \\
\hline Televidente deportivo & $1,23(1,09-1,39)$ & $<0,001$ & $1,34(1,18-1,52)$ & $<0,001$ \\
\hline Lector deportivo & $1,29(1,17-1,44)$ & $<0,001$ & $1,57(1,32-1,88)$ & $<0,001$ \\
\hline \multicolumn{5}{|l|}{ Miembros del hogar con ADR } \\
\hline Jefe del hogar no hace ADR & 1,00 & & 1,00 & \\
\hline Jefe del hogar hace ADR & $1,77(1,60-1,90)$ & $<0,001$ & $2,26(2,06-2,47)$ & 0,010 \\
\hline
\end{tabular}

${ }^{a}$ Actividad deportiva regular, definida como la práctica de algún tipo de deporte con frecuencia diaria o interdiaria durante el último mes (sí = 1, no $=0$ ).

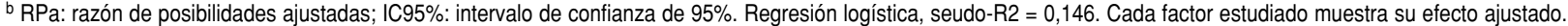

cación ni el nivel socioeconómico mostró una asociación significativa con la ADR. Por otro lado, en ambos sexos se apreció por igual que estar casado, tener empleo, tener acceso a tecnologías modernas de comunicación (Internet o cable) o consumir información deportiva son factores significativa y directamente asociados con la práctica de ADR (cuadro 7).

El lugar de residencia también aparece asociado con la práctica de ADR. Tanto en hombres como en mujeres, vivir en Lima metropolitana se asocia inversa y significativamente con la práctica de ADR. Finalmente, la práctica de ADR por parte de los miembros de un hogar se asocia significativa- mente con la presencia de un jefe del hogar que practica deportes activamente, y esta relación es más fuerte entre las mujeres $(\mathrm{OR}=2,26)$ que entre los hombres $(\mathrm{OR}=1,77)$.

\section{DISCUSIÓN}

En un mundo donde la actividad física ya no es una necesidad para sobrevivir, la práctica de deportes en el tiempo libre se percibe y promueve como una alternativa al sedentarismo. Los resultados de este trabajo indican que solo la décima parte de la población urbana adulta del Perú practica deportes en un grado capaz de generar beneficios para la salud - es decir, diariamente o en días alternos-, definido aquí como ADR. La proporción de personas que practican ADR, sin embargo, podría estar sobreestimada, ya que en la encuesta solo se preguntaba la frecuencia de la práctica de deportes, sin considerar su intensidad y duración.

Los resultados de esta investigación son ligeramente inferiores a los encontrados por otros autores que utilizaron una definición de actividad física similar a la empleada en el presente trabajo. Según investigaciones realizadas en zonas muy disímiles, $16 \%$ de las personas de Bambuí (Minas Gerais, Brasil) y $17 \%$ de la población de Singapur realizan una actividad depor- 
tiva (23, 24). Según Martínez-Ros (25), $17,8 \%$ de la población de Murcia (España) practica una actividad deportiva intensa (por lo menos 2 horas por semana). En los Estados Unidos de América se encontró que $30,8 \%$ de la población adulta realizaba actividades físicas regularmente en su tiempo libre (26), pero su definición de actividad física difiere de la utilizada aquí.

\section{Factores individuales y percepciones asociadas con la práctica del deporte}

La conclusión de que los hombres practicaban significativamente más ADR que las mujeres, con excepción de los grupos de edad entre los 30 y 44 años, coincide en parte con los datos publicados en Estados Unidos (26), España (27) y Finlandia (28), según los cuales los hombres en todas las edades realizan más actividad física que las mujeres. La práctica de deportes más frecuente entre los hombres podría deberse a determinadas normas sociales y culturales. El acmé de ADR en las mujeres ocurre a los 40 años como promedio, es decir, 10 años antes que en los hombres. Además, las mujeres triplicaron aproximadamente la práctica semanal del deporte entre los 15 y los 60 años de edad, mientras que los hombres solo la duplicaron. La afición más temprana de las mujeres adultas jóvenes a la práctica de ADR podría explicarse por razones de imagen corporal o salud y un mayor nivel de información. Sin embargo, este estudio no permite elucidar estas hipótesis, por lo que tendrán que ser tema de trabajos futuros antes de aplicarlas en el diseño de intervenciones.

Según diversos autores, el nivel socioeconómico, los ingresos y el nivel educativo están directamente asociados con la práctica de actividad deportiva recreativa $(25,29-31)$. En el presente estudio solo la educación se mostró significativamente asociada con la práctica de ADR y esta asociación estuvo restringida a los hombres.

Una posible interpretación de la falta de asociación entre el nivel socioeconómico y la actividad física podría ser que la variable "ingresos" no es suficientemente sensible para medir los componentes que podrían estar asociados con la práctica de ADR. En efecto, existen variables que reflejan mejor el nivel económico - como es el caso de estar empleado, tener acceso a cable o Internet- y que aparecen asociadas en sentido directo con la práctica de ADR, tanto en hombres como en mujeres. Los resultados obtenidos en el presente trabajo apoyan esta hipótesis. Este es un aspecto que requiere más estudio y que podría ayudar a explicar las diferencias encontradas entre la práctica de ADR en hombres y mujeres.

Las barreras más importantes para la práctica de deportes percibidas por los jefes de hogar fueron, independientemente del sexo, la falta de tiempo, el desinterés y la falta de infraestructura deportiva. Todas ellas coinciden con observaciones hechas en Estados Unidos (32) y en Inglaterra (33), donde adicionalmente se encontró que esas barreras variaban de acuerdo con la edad y la clase social (33). La percepción de falta de tiempo podría revelar un fenómeno real, como lo indica un estudio sobre factores determinantes de la obesidad realizado en Perú, en el que se encontró que quienes trabajan más de 40 horas por semana tienen $50 \%$ más probabilidades de ser obesos y de realizar menos deportes recreativos que quienes trabajan 20 horas a la semana o menos (9). De manera similar, la percepción de la falta de infraestructura deportiva podría reflejar una realidad. No obstante, las barreras referidas — falta de tiempo y necesidad de espacios de recreaciónpueden ser modificadas mediante intervenciones efectivas en función del costo (34-36).

\section{El ambiente y la ADR}

Vivir en zonas urbanas fuera del área de Lima metropolitana — sobre todo en la selva, la costa centro y la sierra centro- fue un factor fuertemente asociado con la práctica de ADR, independientemente de la percepción del entrevistado. La organización fí- sica, ambiental, social y cultural de ciudades más pequeñas y menos urbanizadas que la capital podría influir en la práctica de ADR. De hecho, se han identificado algunos elementos del desarrollo urbano - como la seguridad en las calles, la criminalidad, la disponibilidad de ambientes de recreo, el transporte público y la contaminación ambiental, entre otros - relacionados con los hábitos de actividad física de la población $(37,38)$.

Es interesante anotar que en los hogares donde el jefe de la familia practicaba ADR, otros miembros de la familia tendían a practicar ADR, hecho que fue más acentuado cuando el jefe del hogar era mujer. Es probable que la conducta del jefe de la familia en relación con el deporte genere una motivación importante para el resto de la familia, que se traduce en una mayor dedicación a la práctica de deportes. De manera similar, un estudio realizado en Australia identificó el ambiente físico, los factores individuales y el entorno social como elementos clave que estimulan la práctica de actividad física en la población (39). Estas dos variables ambientales (características urbanas diferenciadas y entorno socio-familiar) ayudan a explicar la predisposición a la práctica de ADR y deben tenerse en cuenta para el diseño de intervenciones futuras.

Una limitación importante de este estudio es que solo se exploró un aspecto de la actividad física de las personas: el deporte. La inclusión de otras formas de actividad podría ayudar al análisis de otros niveles de actividad física que resulten igualmente beneficiosos para la salud y permitiría revelar otras posibilidades de intervención. Un importante instrumento en esta dirección es el Cuestionario de Actividad Física Internacional (40), desarrollado recientemente, que deberá ser empleado en estudios futuros.

\section{CONCLUSIONES}

Los resultados de este trabajo permiten afirmar que la práctica de ADR en las zonas urbanas del Perú es escasa. Las personas más afectadas son 
las menores de 30 años, las mujeres y los residentes en el área metropolitana de la capital. Esta insuficiente práctica de ADR constituye un reto para la salud pública y, al mismo tiempo, reafirma la necesidad de promover estilos de vida activos, adaptables a la vida cotidiana, que abarquen actividades tales como caminar, bailar o montar bicicleta. Esto podría ayudar a eliminar obstáculos para la práctica del deporte, como son la falta de tiempo y de infraestructura deportiva. La influencia positiva observada cuando el jefe del hogar es un deportista activo y la mayor ADR encontrada en zonas urbanas fuera de la ciudad de Lima deben ser objeto de estudios más profundos y deben tomarse en consideración para el diseño de intervenciones específicas.

\section{REFERENCIAS}

1. Word Health Organization. Feachem RG, Kjellstrom T, Murray CJ. The health of adults in the developing world. Washington, D.C.: World Bank; 1999

2. Martorell R, Khan LK, Hughes ML, GrummerStrawn LM. Obesity in Latin American women and children. J Nutr 1998;128(9):14641473.

3. Peña M, Bacallao J. Obesity among the poor: an emerging problem in Latin America. En: Peña M, Bacallao J, eds. Obesity and poverty: a new public health challenge. Washington, D.C.: PAHO; 2000. Pp. 3-10.

4. Organización Panamericana de la Salud. La salud en las Américas. Vol I. Washington, D.C.: OPS; 1998. (Publicación Científica No. 569).

5. Leigh J. Occupational hazards. En: Murray CJL, Lopez AD, eds. Quantifying global health risks: the burden of disease attributable to selected risk factors. Cambridge: Harvard University Press; 1996.

6. Organización Panamericana de la Salud. Encuesta multicéntrica en siete ciudades de América Latina sobre salud, bienestar y envejecimiento (SABE). Washington, D.C.: OPS; 2000.

7. Perú, Instituto Nacional de Estadística e Informática. Encuesta Nacional de Demografía y Salud (ENDES), 1992 y 1996. Lima: INEI; 1996.

8. Jacoby E. Viejos problemas y nuevas epidemias: el reto de la transición de salud en el Perú. En: Cortez R, ed. Salud, equidad y pobreza en el Perú: teorías y nuevas evidencias. Lima: Centro de Investigación de la Universidad del Pacífico; 2002. Pp. 313-337.

9. Cortez R, Jacoby E, Alarcón G. Determinantes de la obesidad y el sobrepeso en el Perú. En: Cortez R, ed. Salud, equidad y pobreza en el Perú: teoría y nuevas evidencias. Lima: Centro de Investigación de la Universidad del Pacifico; 2002. Pp. 339-375

10. Pate R, Pratt M, Blair S, Haskell WL, Macera CA, Bouchard CA, et al. Physical activity and public health: a recommendation from the Centers for Disease Control and Prevention and the American College of Sports Medicine. JAMA 1995;273:402-407.

11. Physical activity and health: a report of the Surgeon General. Atlanta, Georgia, USA: CDC; 1996.

12. Rich-Edwards J, Manson J, Hennekens, Buring $\mathrm{J}$. The primary prevention of coronary heart disease in women. N Engl J Med 1995; 322:1758-1766.
13. Kilander L, Berglund L, Boberg M, Vessby B and Lithell $\mathrm{H}$. Education, lifestyle factors and mortality from cardiovascular disease and cancer. A 25-year follow-up of Swedish 50year-old men. Int J Epidemiol 2001;30:11191126.

14. Manson JE, Rimm EB, Stampfer MJ, Colditz GA, Willet WC, Krolewski AS, et al. Physical activity and incidence on non-insulindependent diabetes mellitus in women. Lancet 1991;338:774-778.

15. Tuomilehto J, Lindstrom J, Eriksson J, Valle TT, Hamalainen H, Ilanne-Parikka P, et al. Prevention of type 2 diabetes mellitus by changes in lifestyle among subjects with impaired glucose tolerance. N Engl J Med 2001; 344:1343-1350.

16. Bronner L, Kanter D, Manson J. Primary prevention of stroke. N Engl J Med 1995;333: 1392-1400.

17. Rosenbaun M, Leibel R, Hirsch J. Obesity. N Engl J Med 1997;337:396-407.

18. Snow-Harter C, Marcus R. Exercise, bone mineral density, and osteoporosis. Exerc Sport Sci Rev 1991;19:351-388.

19. Byrne A, Byrne DG. The effect of exercise on depression, anxiety and other states: a review. J Psychosom Res 1993;37:565-574.

20. Luoto R; Latikka P, Pukkala E, Hakulinen T, Vihko V. The effect of physical activity on breast cancer risk: a cohort study of 30,548 women. Eur J Epidemiol 2000;16:973-980.

21. Bouchard C. Physical activity and health: introduction to the dose-response symposium. Med Sci Sports Exerc Suppl 2001:33(6):347-350.

22. Instituto Cuánto, Banco Mundial. Encuesta nacional de niveles de vida. Lima: Instituto Cuánto; 2000

23. Lima-Costa M, Barreto S, Uchoa E, Firmo J Vidigal P, Guerra E. The Bambuí Health and Aging Study (BHAS): prevalence of risk factors and use of preventive health care services. Rev Panam Salud Publica 2001;9(4): 219-227.

24. Cutter J, Yian TB, Kai Chew S. Levels of cardiovascular disease risk factors in Singapore following a national intervention programme. Bull WHO 2001;79(10):908-915.

25. Martínez-Ros MT, Tormo MJ, Pérez-Flores D, Navarro C. Actividad física deportiva en una muestra representativa de la población de la región de Murcia. Gac Sanit 2003:17(1):11-19.

26. Crespo C, Smith E, Andersen R, Carter-Prokas $\mathrm{O}$, Aimsworth BE. Race/ethnicity, social class and their relation to physical inactivity during leisure time: results from the third national health and nutrition examination survey, 1988-1994. Am J Prev Med 2000;18(1):46-53.

27. Domínguez-Berjón $M$, Borrell $C$, Nebot $M$, Artazcoz L, Moncada S, Plasencia A. La actividad física de ocio y su asociación con variables sociodemográficas y otros comportamientos relacionados con la salud. Gac Sanit 1998;12(3):100-109.

28. Telama R, Yang X. Decline of physical activity from youth to adulthood in Finland. Med Sci Sports Exerc 2000;32(9):1617-1622.

29. Droomers M, Schrijvers C, Van der Mheen $H$, Mackenbach JP. Educational differences in leisure-time physical activity: a descriptive and explanatory study. Soc Sci Med 1998;47: 1665-1676.

30. Droomers M, Schijvers C, Mackenbach JP. Educational level and decreases in leisure time physical activity: predictors from the longitudinal GLOBE study. J Epidemiol Community Health 2001;55:562-568.

31. Brownson R, Eyler A, King A, Brown DR, Shyu YL, Sallis JF. Patterns and correlates of physical activity among US women 40 years and older. Am J Public Health 2000;90:264270.

32. Chinn D, White M, Harland J, Drinkwater C, Raybould S. Barriers to physical activity and socioeconomic position: implications for health promotion. J Epidemiol Community Health 1999;53:191-192.

33. West P, Reeder A, Milne B, Poluton R. Worlds apart: a comparison between physical activities among youth in Glasgow, Scotland, and Dunedin, New Zealand. Soc Sci Med 2002; 54:607-619.

34. Browson RC, Houseman R, Brown DR, JacksonThompson J, King AC, Malon BR, et al. Promoting physical activity in rural communities: walking trail access, use and effects. Am J Prev Med 2000;18:235-241.

35. Kahn EB, Ramsey LT, Brownson RC, Heath GW, Howze EH, Powell KE, et al. and the Task Force on Community Preventive Services. The effectiveness of interventions to increase physical activity: a systematic review. Am J Prev Med 2002;22:73-107.

36. Sallis JF, Bauman A, Pratt M. Environmental and policy interventions to promote physical activity. Am J Prev Med 1998;15(4):379-396.

37. Humpel N, Owen N, Leslie E. Environmental factors associated with adults' participation in physical activity. Am J Prev Med 2002;22(3): 188-199. 
38. French S, Story M, Jeffery R. Environmental influences on eating and physical activity. Ann Rev Public Health 2001;22:309-335.

39. Giles-Corti B, Donovan R. The relative influence of individual, social and physical en- vironment determinants of physical activity. Soc Sci Med 2002;54(12):1793-1812.

40. International Physical Activity Questionnaire. [Sitio en Internet]. Disponible en: http://www. ipaq.ki.se. Acceso el 10 de setiembre de 2002.
Manuscrito recibido el 11 de diciembre de 2002. Aceptado para publicación, tras revisión, el 5 de junio de 2003

ABSTRACT Objectives. To determine the frequency of sports physical activity in the urban population of Peru and to identify the sociodemographic, economic, and environmental factors associated with that activity.

Sociodemographic and environmental factors associated with sports physical activity in the urban population of Peru
Methods. This study utilized information collected by the country's National Household Survey (Encuesta Nacional de Hogares) in the second quarter of 1997. That Survey is overseen by Peru's National Institute of Statistics and Informatics (Instituto Nacional de Estadística e Informática). The Survey was based on a probabilistic, multistage sample that was stratified for all the urban areas of the country, which was divided into eight geographic regions: metropolitan Lima, northern coast, central coast, southern coast, northern mountains, central mountains, southern mountains, and jungle. In total, 14913 homes were visited and 45319 people at least 15 years of age were interviewed. The frequency of engaging in sports physical activity was classified as daily, every other day, weekly, or occasional. "Regular sports activity" (RSA) was defined as engaging in sports either every day or every other day. The preferences for and obstacles to sports practice were also examined. A descriptive analysis of the levels of RSA was carried out for gender, using the chi-square test. The factors associated with RSA were analyzed through conditional multiple logistic regression and analysis of residuals, multicollinearity, and interactions. The level of significance was set at $P<0.05$.

Results. Practicing sports at least once a week was more common among men $(44.5 \%)$ than among women $(32.4 \%)$, and the same was true for RSA (12.8\% versus $10.5 \%)$. The age group with the highest level of RSA was 50-55 years for men (20\%), and 40-45 years for women $(18 \%)$. RSA was most common in three geographic regions: jungle $(15.3 \%)$, central mountains (12.8\%), and central coast (12.1\%). RSA was least common in two regions: southern mountains (9.7\%) and metropolitan Lima (10.6\%). The income bracket was not associated with RSA. However, other variables associated indirectly with the socioeconomic level-such as having more formal education, being employed, and having access to the Internet or cable television-and consumption of sports information were significantly and directly associated with RSA. The most frequent barriers to practicing sports were the lack of time, the lack of nearby sports infrastructure (playing fields or courts, etc.), and people's lack of interest. RSA on the part of the members of a household was significantly associated with RSA performed by the head of the household (male or female).

Conclusions. RSA is limited in the urban areas of Peru. This is most true for persons who are less than 30 years old, for women, and for residents of the Lima metropolitan area. This low RSA level is a challenge for public health, and it confirms the need for promoting active lifestyles. More study is needed on the observed positive influence when the head of the household performs RSA and on the fact that RSA is more common in urban areas outside metropolitan Lima. These two findings should also be taken into consideration in designing specific interventions. 